\title{
Design of Power Over Wi-Fi Rectifier Circuit for IoT Based Sensor Application through Simulation
}

\author{
Aiman Saifullah Aini Azman ${ }^{1}$ and Nor Azuana Ramli ${ }^{2 *}$ \\ ${ }^{1}$ Electrical Engineering Section, Universiti Kuala Lumpur British Malaysian Institute, Gombak, Selangor, \\ Malaysia. \\ $2^{2 *}$ Centre for Mathematical Sciences, Universiti Malaysia Pahang, 26300, Gambang, Pahang, Malaysia \\ *Email: azuana@ump.edu.my
}

\begin{abstract}
Energy harvesting has been a phenomenon throughout the year as it produces renewable clean energy which not only benefits the user but also the environment. Radiofrequency energy is among the possible choice that can be harvested for low power usage applications. By doing so, low power applications such as IoT-based sensors can eliminate the usage of input power from the battery which has limited supply over time or restrained from limited cable length. The objectives of this study are to design a power over Wi-Fi rectifier circuit for IoT-based sensor application, analyze the effect of different types of diodes on the power over Wi-Fi rectifier circuit, and determine of minimum voltage required to power an IoT sensor. Cockcroft Walton voltage multiplier configuration was chosen as the rectifier circuit to be employed in the simulation by using Multisim software. With two different Schottky diodes, the output was recorded and analyzed. Both diodes have achieved the minimum required voltage of 3.3V and above for IoT sensors at stage 3. Among the diodes, BAT68 has recorded better output with $4.145 \mathrm{~V}$ compare to diode BAT17 with $4.090 \mathrm{~V}$. The output of diode BAT68 also has reached its stable condition fast than diode BAT17. This configuration can be used and applied in prototypes for future study. Even though many other factors need to be considered, radiofrequency energy harvesting should be explored more as it promotes clean energy and longevity lifespan as the world is moving wireless in many tasks.
\end{abstract}

Indexed Terms- energy harvesting, rectifier circuit, power over Wi-Fi, Multisim, clean energy

\section{INTRODUCTION}

As the world today is full of technologies connecting our gadgets and accessories, be it home appliances, medical check-ups, payment methods, and many more, it is not uncommon that our buildings were destined for the same fate. In Malaysia, since Multimedia Super Corridor (MSC) was introduced by the government in 1996, numerous intelligent or smart buildings have been built over the years. Uses of a set of sensors built in the infrastructure and Internet of Things (IoT) allows a significant improvement in the management of smart buildings. However, despite the uses of normal IoT-based sensors, there is still improvement that can be made to utilize and maximize the potential of the sensors.

The problem with the existing IoT-based sensor is most of the sensors required input sources from the power outlet or battery to power up. Standard operating voltage for these IoT sensors is in the range up until $3 \mathrm{~V}$ and many of the industry have been using $3 \mathrm{~V}$ coin batteries which are undeniably cheap and reliable. However, such batteries need to be replaced quite frequently. A fully charged lithium battery supplies approximately $3.2 \mathrm{~V}$ and the voltage drop to $3 \mathrm{~V}$ after operating for few hours, which might not enough to power up certain devices for a convinced period.

Besides that, some of the installations of IoT-based sensors require to be placed at an unreachable area which makes it difficult for the installer or the person in charge. For a smart building management system, multiple types of sensors are expected to be used to fulfill the objective itself which uses automated processes that automatically control the building operation to increase people's comfort while providing solutions in analyzing and improving the energy efficiency, and reduce the 
consumption and costing. The expected sensors can be ranging from temperature, humidity, lux, voltage, current, power and energy sensor. The difficulties to install these IoT sensors should have been tackled instantly as the application is widely been used today. This is where the application of power over wifi will come in handy as it will provide and enable these IoT sensors the mobility to be installed freely no matter the condition of the area.

The power over wi-fi (PoWiFi) system had been developed by a team of researchers and engineers from the University of Washington in 2015 where the technology is used to power up various devices by using a Wi-Fi router [1]. Despite having the lowest frequency range among the other radio frequency (RF) sources as listed in Table 1, Wi-Fi signals are chosen as their relative ease to access across various places. It grants a good platform to develop the PoWiFi signals to power up the IoT sensor. The concept has been proved by the researchers from the University of Washington by building the battery-free and battery-recharging harvester for a temperature sensor and an image sensor. Hence, this study aims to develop a PoWiFi rectifier circuit for the IoT-based sensor. To achieve the objective, a suitable methodology is proposed which will be discussed briefly in the next section after a discussion on some previous related studies.

Table 1: Frequency Range for Various RF Sources [2]

\begin{tabular}{ll}
\hline RF Source & Frequency Range \\
\hline TV Transmission & $54-806 \mathrm{MHz}$ \\
FM Transmission & $88.1-108.1 \mathrm{MHz}$ \\
Global System for Mobile Communication & $902-928 \mathrm{MHz}$ \\
Wi-Fi & $2.40-2.484 \mathrm{GHz}$ \\
\hline
\end{tabular}

\section{LITERATURE REVIEW}

Energy harvesting or energy scavenging is a process of energy conversion technique employed for converting energy from the ambient atmosphere into useful electrical energy. The term energy harvesting is frequently applied to provide a supply source to power up various wireless applications. Power over Wi-Fi is a system that targets specifically only the Wi-Fi signal. According to Lenord Melvix et al. [1], RF energy delivers the highest efficiency power harvested compared to other sources which at $33 \%$. The ability of harvesting this energy will enable continuous charging low power devices, hence, eliminate the need for a battery. These devices can be free from connectors, cables, and battery charge ports. This method has become more popular over the years as the ability to access Wi-Fi is everywhere and the idea can be implemented in many applications essentially for low power applications. Based on the previous study by Lenord Melvix et al. [1] a prototype of an RF energy harvester has been designed specifically for low power RFID tag application.

Radiofrequency energy harvesting methods are often called rectifying antenna or rectenna. A rectenna is a vital component to develop power over Wi-Fi system. It is an integral of an antenna and rectifier to collect RF energy from the surrounding and convert it to DC voltage. There are numerous designs of rectennas have been developed throughout the studies. A rectenna proposed by Shafique et al. [3] consists of a receiving antenna, a bandpass filter, a matching circuit, a rectifying diode, and a low pass filter. The AC induced in the antenna will be rectified by the diode to produce DC power. Figure 1 shows the basic schematic structure of a simple rectenna circuit. 


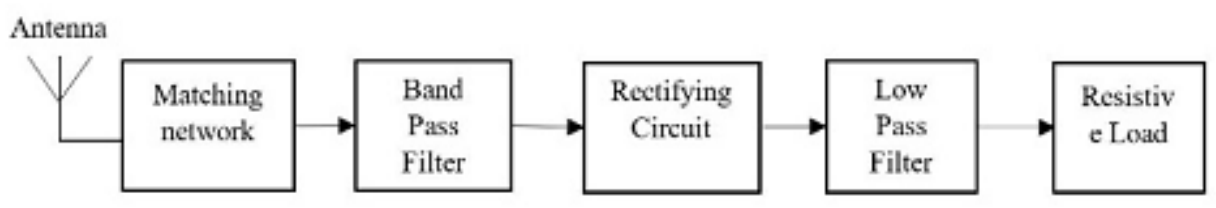

Figure 1: Schematic of rectenna structure [4]

An antenna is used to collect the RF energy in the surrounding at a designated frequency. Next, the power received will further move to the matching circuit. A matching circuit here will maximize the power to be transferred to the rectifier. This energy then will pass through to the bandpass filter. Noise and unwanted frequency components will be filtered out by the pass filter. For the rectification process, the diode used needs to have the lowest voltage drop and highest speed so the power losses due to conduction and switching are minimal. This is because the Schottky diode was chosen. A low pass filter is the last step in the rectenna circuit before being transferred to the resistive load. The purpose is to smoothen the signal and remove any noise.

Since this study focuses on the output voltage from the type of diode employed and the power over Wi-Fi rectifier circuit to power up the wireless sensor, the methods from previous studies are very crucial for references. The deployment of the method used in rectenna from previous studies has been compared. The antennas that have been deployed by all the researchers are different from each other. Olgun et al. [4] chose to use a Kock patch antenna but in an array, which means there are multiple antennae used. Lenord Melvix et al. [1] chose to use a single hybrid Kock Meander monopole antenna while Thosar [5] chose to use a microstrip patch antenna. The antenna is one of the crucial parts as it plays the role where the energy is scavenged. However, these three antennas type that has been deployed in the previous studies shared the same purpose which was to maximize their input gained and reduce the size and cost.

As for the frequency, RF harvesting is not limited to $2.4 \mathrm{GHz}$. All ranges of frequency can be scavenged depending on the antenna design and parameter. Rosli et al. [6] in their study chose to simulate their radio frequency harvesting method by using $900 \mathrm{MHz}$ to $2.4 \mathrm{GHz}$. The frequency of 900 $\mathrm{MHz}$ produced higher output voltage with differences not more than $10 \mathrm{mV}$ compare to $2.4 \mathrm{GHz}$. All of these studies chose a $2.4 \mathrm{GHz}$ frequency band. This is because the aim of those researches must be targeting to scavenge the radio frequency energy from Wi-Fi. So, the frequency band chosen is depending on the objective of the study and to harvest RF energy from which source. It can be $900 \mathrm{MHz}$ which the input source usually comes from mobile phone GSM application or $2.4 \mathrm{GHz}$ which came from the microwave, Bluetooth, or wireless data transfer application.

Moving on to the rectifier circuit, all these three studies in [1], [5], and [6] deployed different methods of rectifying. Greinacher and Cockcroft Walton configuration is the common method used in RF harvesting study. However, all these methods lead to the same purpose which to convert RF energy that comes in AC form to DC form. Other than that, the purpose of the rectifier is to amplify the input voltage because the input usually is too small. Nevertheless, the amplification part can also be done by using other methods such as DC booster or Charge Pump which has been done by Bandyopadhyay and Dutta [7]. Back to the rectifier, a comparison between output produce by Greinacher and CockcroftWalton configuration was done [1]. The result was Cockcroft-Walton multiplier produced higher output DC voltage compared to the Greinacher rectifier. However, harmonic suppression achieved in Greinacher configuration produce is better compared to another one. Aside from that, all these configurations need to be done in multiple stages to achieve their desired minimum voltage.

Latest study related to current transducer (CT) for IoT applications had been done by Bonaldi et al. [8]. This study presents one of these evolutions showing a CT, which can measure this magnitude, make an initial treatment of the signal, and transmit it to a panel or control center. There are other advantages of the CT had been highlighted in the study such as the CT does not require an energy source to operate as it has self-powered by the current and it is inexpensive. With all these advantages and signal treatment, $\mathrm{CT}$ is proposed to be a part of the world of IoT. 


\section{RESEARCH METHODOLOGY}

Comprehensive researches had been made before the PoWiFi and its working mechanism is developed. This is to ensure the proper approach can produce the desire output and objective of the study can be achieved at the end of the study. A literature review was the first step made for more understanding of the radiofrequency energy harvesting concept and method. Different studies used different approaches to optimize the energy harvested and to achieve their desired output. This research then is continued by selecting the most convenient method to be used for rectenna design. This is the most crucial part as the selection method will affect the whole process and outcome.

Rectenna are an integral part of a rectifier and an antenna to amass the wireless RF energy from free space and convert it to a DC voltage. Usually, it consists of a receiving antenna, an impedance matching network, a rectifying diode, and a DC bypass filter. An antenna is used to capture the RF energy in the designated frequency. The energy then will pass through the impedance matching circuit to match the diode impedance to the antenna input impedance at the chosen frequency. This is to deliver maximum output power.

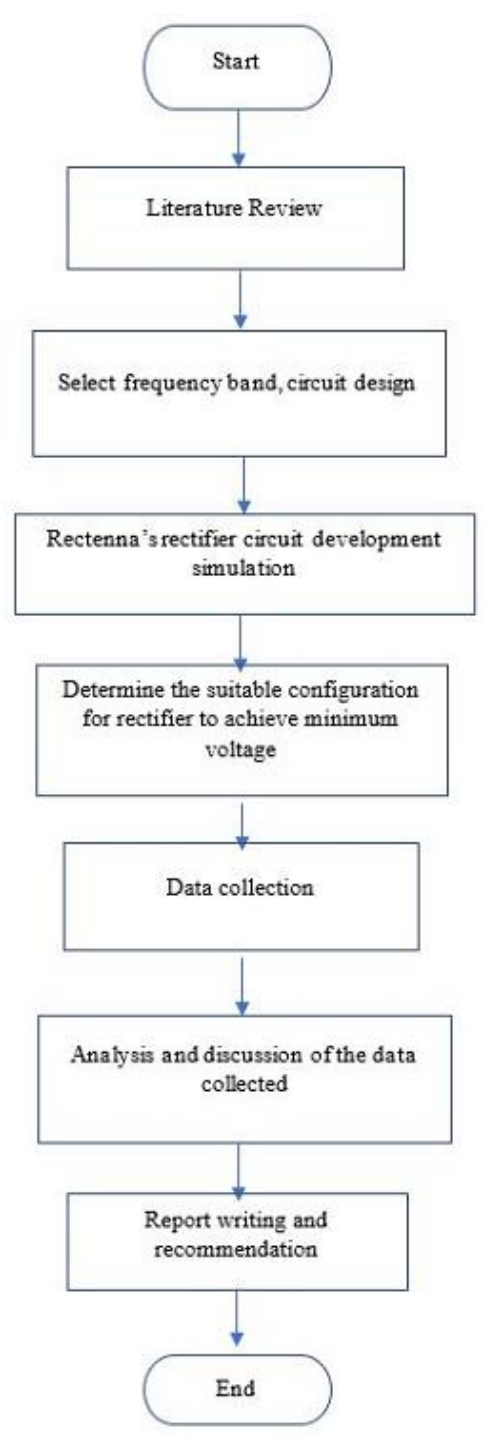

Figure 2: A research framework 
Next, the selection of rectifier diode configuration where the conversion process occurred here and the conversion involving the RF energy to DC voltage. After selecting the best approach and configuration of the rectifier for the rectenna component, the data were collected. Analysis and discussion on the data collection will be made before this study end with report writing and recommendations for future study. The whole process for this study is summarized as in figure 2 above while figure 3 shows the process flow of the rectenna's rectifier circuit development to achieve minimum voltage for the wireless sensors.

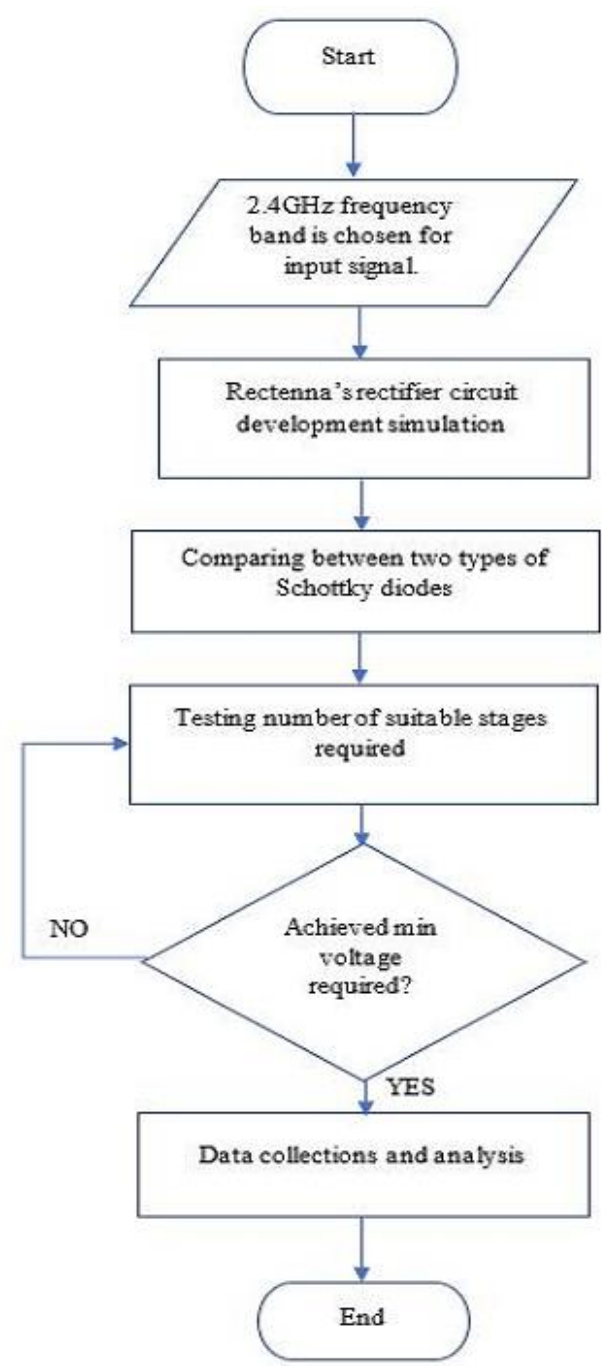

Figure 3: Design process

The process starts with a collection of RF energy by the antenna. The chosen frequency band for the Wi-Fi signal is $2.4 \mathrm{GHz}$. Any other frequency band will not be collected as the antenna is only operated at $2.4 \mathrm{GHz}$. This is because the main objective is to harvest the Wi-Fi energy lingering in an open space inside an office. The process then is continued by developing the rectifier circuit.

The components used are crucial here as they will be influenced by the output voltage later. The example of components that need to be determined here is a diode, capacitor, resistor, and input. However, this stage is only limited to simulation purposes. Under some circumstances, the variation of components chosen is also limited to the components that are available in the software and can be obtained in the Malaysian market at a reasonable price. This is because to close the gap of realization in making the prototype in the future study. 
The process will continue with measuring the output voltage from the rectifier circuit. The output however will be determined from cascading stages of the rectifier. Analysis and discussion will be made afterward from the voltage output collected.

\subsection{Mathematical characteristics}

Radiofrequency (RF) is the wavering pace of an alternating electric flow or voltage or an electric or electromagnetic field. It involves the application of transmission lines, waveguides, and antennas. The value of frequency can be calculated by the formula below:

$f=\frac{c}{\lambda}$

where $c$ is the speed of light, $c=299,792,458 \mathrm{~m} / \mathrm{s}$,

$\lambda$ is the wavelength,

$f$ is the frequency value.

From the formula above, the lower the frequency will result in the larger the wavelength or bandwidth, and the larger the bandwidth the impact on the antenna is higher. The power collected also is higher for a lower frequency compared to a higher frequency. The power in RF application usually will be measured in milliwatt $(\mathrm{mW})$ or decibel milliwatt $(\mathrm{dBm}) . \mathrm{dBm}$ is a unit used to express in decibel $(\mathrm{dB})$ with reference to one $\mathrm{mW}$. The value of $\mathrm{dBm}$ can be calculated by using the formula below. For a 2.4 $\mathrm{GHz} \mathrm{WiFi} / \mathrm{ISM}$ band, the power transmitted from it can go up to $100 \mathrm{~mW}$ or $20 \mathrm{dBm}$.

$\mathrm{dBm}=10 \log _{10} \frac{P}{1 \mathrm{~mW}}$

where $\mathrm{dBm}$ is the absolute power level,

$P$ is the power in Watt,

$1 \mathrm{~mW}$ is one milliwatt.

\subsection{Components in rectenna}

Many types of an antenna can be used in rectenna. Examples of the antenna are dipole, Yagi Uda antenna, microstrip antenna, coplanar antenna, spiral antenna, and many more. Some criteria need to be considered when choosing the antenna such as the frequency band operated, radiation pattern, and impedance. Throughout previous studies, the microstrip antenna is the most selected antenna chosen for the studies. That is because a microstrip antenna is easy to fabricate, less complex, and low cost. Since this study will only employ the simulation, the function generator will assist the antenna role as the input source to the circuit. This method has been employed by [7] and [9] in their previous study.

Same to the antenna, a rectifier is one of the indispensable components in a rectenna. It acts as the converter from RF energy to DC energy. Often for the rectifier in rectenna, Schottky diode is chosen because it has a low forward voltage but a very fast switching action. These lower voltage drops contribute to the higher switching speed and provide better system accuracy. Schottky diode HSMS2850 is chosen in most of the study due to its very small forward voltage, which is $150 \mathrm{mV}$ with $1 \mathrm{~mA}$ forward current, however, the availability of the diode is difficult and pricey. So, in this study, two Schottky diodes that can be acquired from the local market are chosen. These diodes are BAT68 and BAT17. Besides, the forward voltage of these diodes is not as low as the HSMS-2850. The forward voltage for BAT68 is $410 \mathrm{mV}$ and BAT17 is $550 \mathrm{mV}$. The forward current also has a significant difference compared to the HSMS-2850. 
Table 2: Schottky Diode Parameters

\begin{tabular}{|l|l|l|}
\hline Diode & Forward Voltage & Forward Current \\
\hline BAT17 & $550 \mathrm{mV}$ & $130 \mathrm{~mA}$ \\
\hline BAT68 & $410 \mathrm{mV}$ & $130 \mathrm{~mA}$ \\
\hline
\end{tabular}

A rectifier is employed in this study because it acts as the most crucial part of a rectenna which is the conversion of RF energy to electrical energy in form of direct current. A designated type of rectifier circuit that can potentially produce output voltage many times greater than the input voltage applied on the other hand is called voltage multiplier. Various types of the multiplier can be employed for rectenna such as Dickson multiplier, Greinacher multiplier, and Cockcroft-Walton multiplier. In the case of harvesting RF energy, the energy collected at the antenna port to be used as input is known to be very small, which varies around $100 \mathrm{~mW}$ for a $2.4 \mathrm{GHz}$ frequency band. With aid of a voltage multiplier, the output will be step up to meet the minimum required input voltage for the sensors or devices. For this study, the Cockcroft-Walton multiplier will be chosen as the voltage multiplier. This is because a comparison study has been done by Lenord Melvix et al. [1] resulted in that the Cockcroft-Walton multiplier recorded higher output voltage compare to Greinacher but at the expense of lower harmonic.

\section{RESULTS AND DISCUSSION}

\subsection{Input source of radiofrequency energy}

Energy harvesting is a process of energy conversion technique employed for capture and converting the energy in surround into electrical energy. In this study, the value of input will be assumed and manually set. $10 \mathrm{dBm}$ is chosen to be set as the value of the power source. The voltage peak corresponding to the $\mathrm{dBm}$ will be used in the input source of the function generator with a frequency of $2.4 \mathrm{GHz}$. The conversion method is calculated by using the formula below:

$\mathrm{dBm}=10 \log _{10} \frac{V^{2}}{R \times 10^{-3}}$

where $\mathrm{dBm}$ is decibel per milliwatt,

$R$ is the load impedance.

In this formula, $R$ is the load impedance and typically the value is $50 \mathrm{ohms}$ for an RF system. The parameter that needs to be found is voltage. However, the voltage in this formula is the $V_{\text {rms }}$ while the voltage needed in the function generator is $\mathrm{V}_{\text {peak. }}$. To convert $\mathrm{V}_{\text {rms }}$ to $\mathrm{V}_{\text {peak }}$, the formula is as in formula 4.

$\mathrm{V}_{\text {peak }}=\mathrm{V}_{\mathrm{rms}} / 0.707$

In order to study the output voltage generated by the rectifier circuit, the function generator is set to sinusoidal waveform and the voltage peak is set at $1 \mathrm{~V}$. This result is only applied to this parameter and in the simulation by using Multisim only as many situations and parameters such as the distance of rectifier from the input source and the S-parameter of the antenna are not being touched in this study.

\subsection{Simulation}

The simulation was performed in the Cockcroft Walton voltage multiplier configuration. For the starter, the simulation was done in one stage. The circuit consists of one function generator that provides input in a monotone stationary state, two capacitors, two Schottky diodes, one resistor which is set to 100 
$\mathrm{kOhms}$ and acts as the load, connected to a multimeter and oscilloscope. These two circuits contain two different models of Schottky diode.

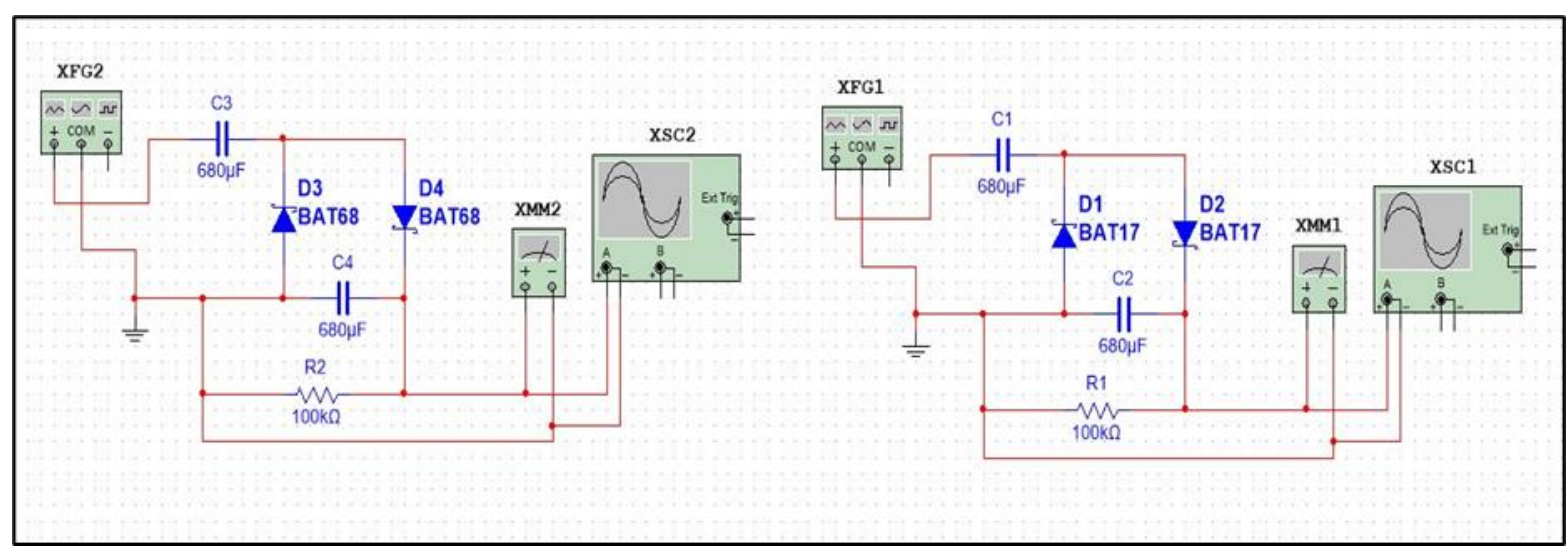

Figure 4: One stage Cockcroft-Walton voltage multiplier with Schottky diode BAT68 and BAT17

The simulation then has been continued by adding a stage to the voltage multiplier. The other components' characteristics and value remained constant. The value of the capacitors in the second stage however is different from the first stage. The capacitors used in the second stage are lower than in the first stage. This method is repeated until the Cockcroft Walton voltage multiplier reaches four-stage.

\subsection{Comparison between stages}

Based on both figure 5 and figure 6 , all the output voltage recorded from each stage can be seen were increasing over time until they reach a certain peak for a period or duration of 20 seconds and more.

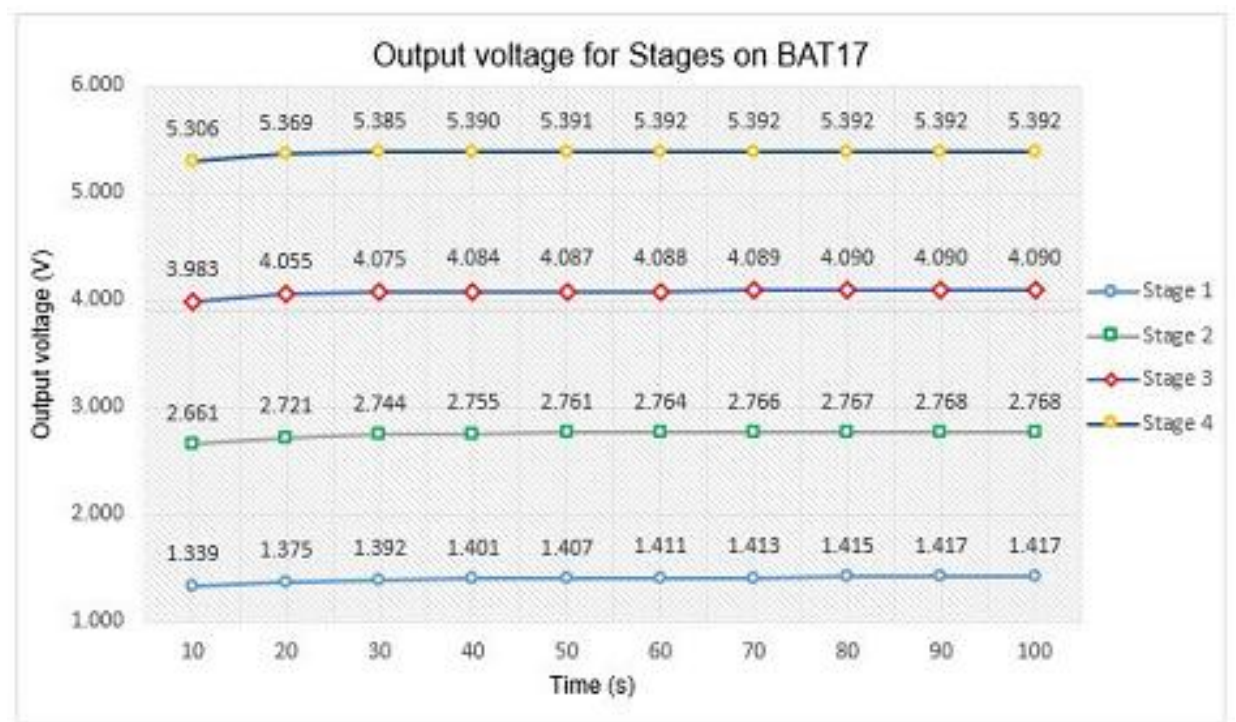

Figure 5: Graph of output voltage for BAT17

By looking at stage one and stage three of diode BAT17 as an example, the time taken for stage one to reach its final output voltage was at the 90 seconds while the time taken for stage three to reach its final output voltage was 80 seconds. Meanwhile, at stage four, the time taken for it to reach its final output voltage was at 60 seconds. 


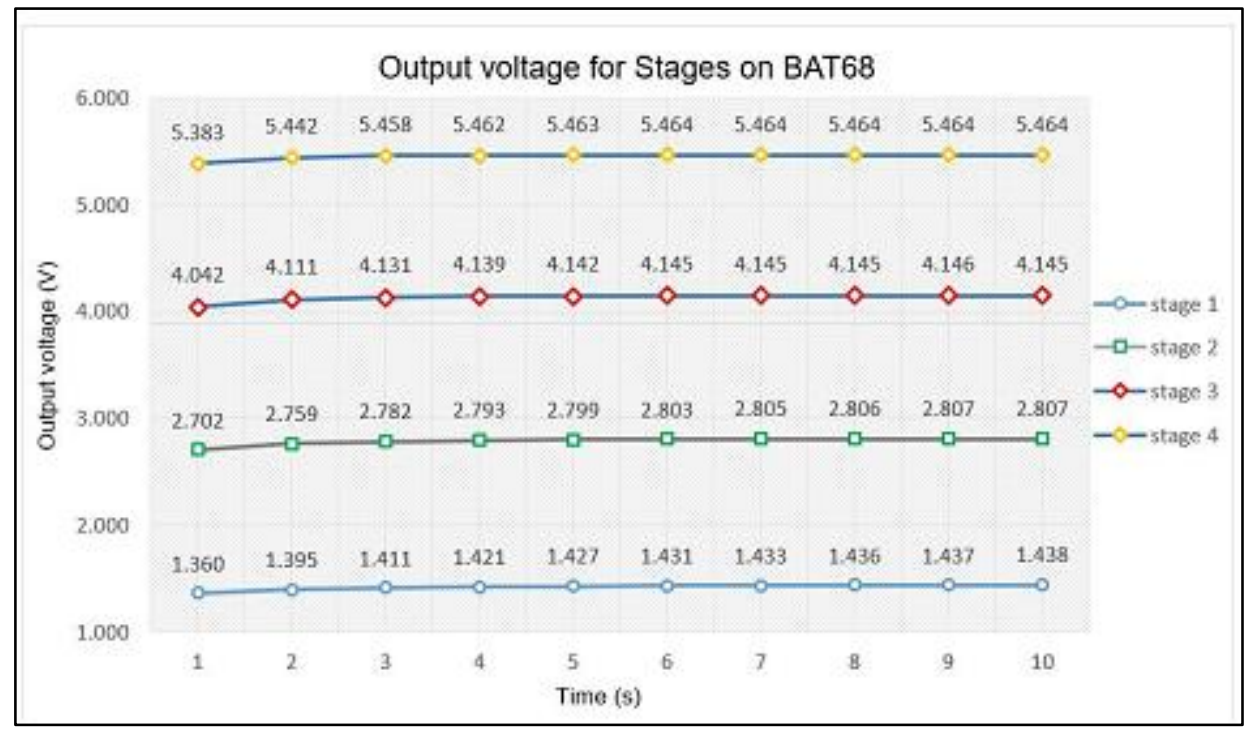

Figure 6: Graph of output for BAT68

Same goes for stage two and stage four diode BAT68, at stage two the final output voltage was recorded to remain the same at the time of 90 seconds while the output voltage remains the same on stage four at the time of 60 seconds. As the stages increase, the time for the output voltage to reach its limit value was decreasing. It can be concluded that the time for the rectifier to reach its final output is shorten when the stages increase. By adjudging from both graphs in Figures 5 and 6, output voltage recorded from diode BAT68 is higher than output voltage recorded from diode BAT17. This can be seen as applicable on all stages. For stage one, the difference between these two diodes is $0.021 \mathrm{~V}$, where BAT68 final output was $1.438 \mathrm{~V}$ while BAT17 final output was 1.417 . For stage two, the difference recorded between the diodes is $0.039 \mathrm{~V}$ with the final output of $2.807 \mathrm{~V}$ from BAT68 and $2.768 \mathrm{~V}$ from BAT17. At stage three, the difference between diode BAT68 and BAT17 is $0.055 \mathrm{~V}$ with the final output of $4.145 \mathrm{~V}$ from BAT68 and $4.090 \mathrm{~V}$ from BAT17. For stage four, BAT68 final output was $5.464 \mathrm{~V}$ and BAT17 recorded its final output at $5.392 \mathrm{~V}$, resulting in the difference between this two on this stage at $0.0072 \mathrm{~V}$.

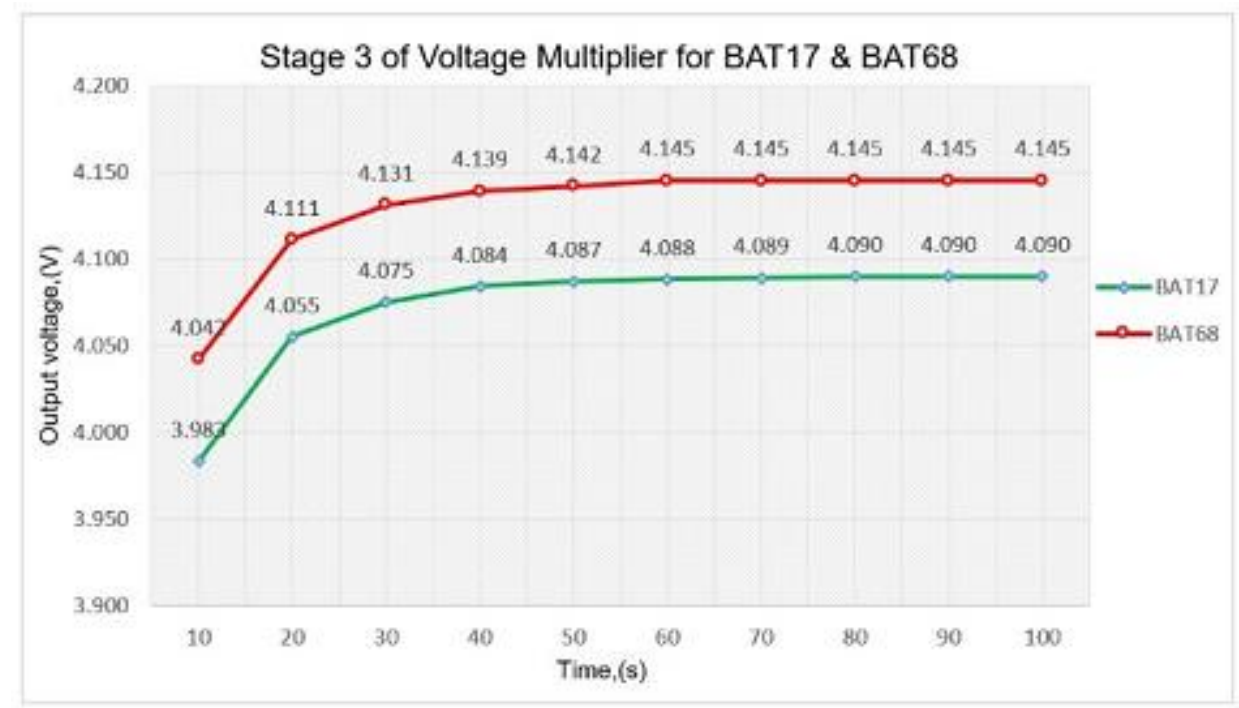

Figure 7: Graph comparison of stage 3 between BAT17 and BAT68 
From this observation, both diodes have been recorded and reach a promising output to meet the aim of this study which to power up an IoT-based sensor that was recorded at stage three. The graph in figure 7 shows a comparison of output voltage between both diodes at stage three for a clearer view. For diode BAT17, the first 10 seconds recorded $3.383 \mathrm{~V}$ and ends at $4.090 \mathrm{~V}$ at 80 seconds. Meanwhile, diode BAT68 recorded $4.042 \mathrm{~V}$ for its first 10 seconds and ends at $4.145 \mathrm{~V}$ at 60 seconds. So, it is safe to say that the minimum required voltage for an IoT-based sensor to operate is at stage three of the voltage multiplier.

Schottky diode BAT68 recorded way higher output voltage than BAT17 at all time as shown in figure 8. The time taken for BAT68 to reach its maximum final output also is faster than BAT17 by 20 seconds. This clearly shows that Schottky diode BAT68 is more suitable to be used in radio frequency harvesting rectifier circuits compare to BAT17. However, this outcome is already expected because of the difference in forwarding voltage of the Schottky diodes. BAT68 has a lower value of forwarding voltage compare to BAT17, thus increase the time for the rectification process and provide better rectification

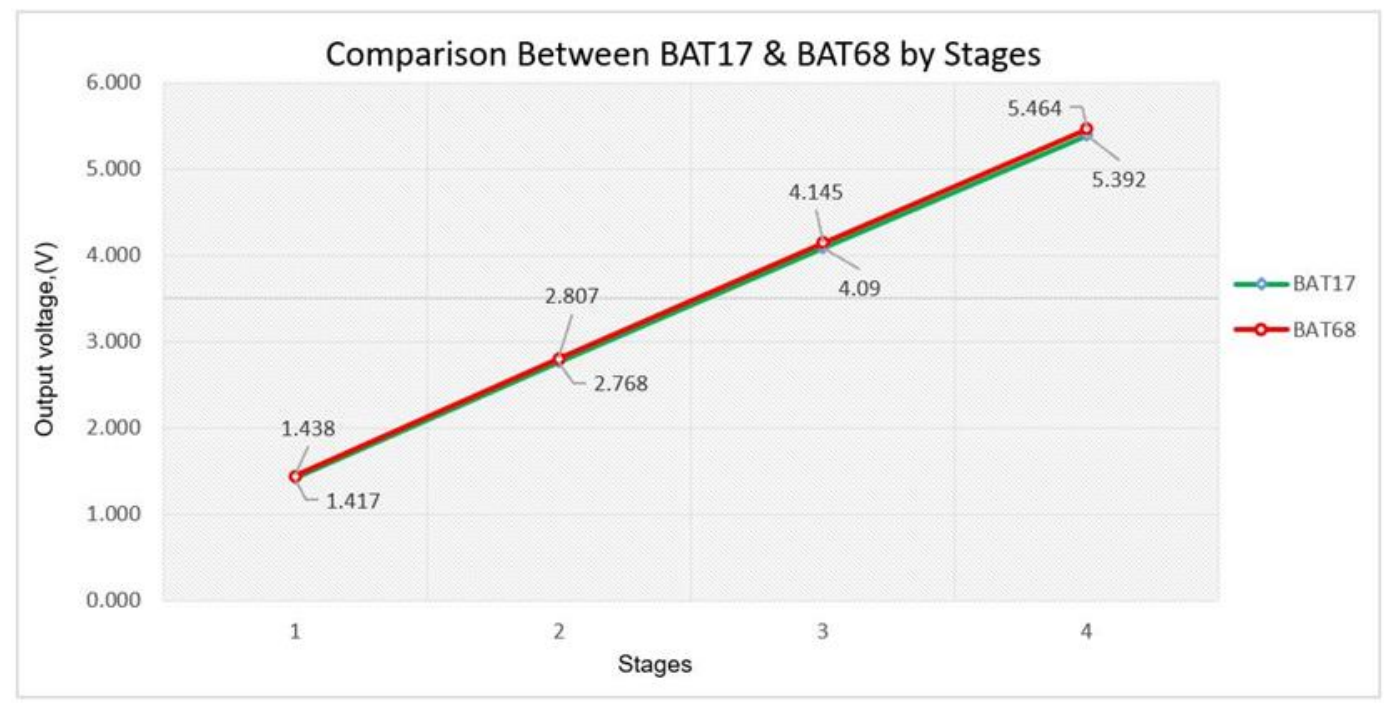

Figure 8: Graph comparison between BAT17 and BAT68 by stages

\subsection{Comparison with the previous study}

A comparison between the previous study and the present study has been done and presented in Table 3. The study that has been chosen for the comparison was by Lenord Melvix et al. [1]. To add, the only simulation was done by both studies.

Table 3: Comparison of the present with the previous study

\begin{tabular}{|l|l|l|}
\hline Research & Previous study & Present study \\
\hline Type of antenna & Hybrid Kock Meander monopole antenna & None \\
\hline Frequency band & $2.45 \mathrm{GHz}$ & $2.4 \mathrm{GHz}$ \\
\hline Method & 5 stages of Cockcroft Walton & 3 stages of Cockcroft-Walton \\
\hline Diode & HSMS2860 & BAT68 and BAT17 \\
\hline Output (Voltage) & $2.058 \mathrm{~V}$ & $4.14 \mathrm{~V}$ and 4.090 V \\
\hline
\end{tabular}

Several components have been compared in Table 3 such as the type of antenna used, frequency band, rectifier method, diode, and final output voltage. By looking straight at the output voltage 
produced from both studies, the present study recorded $4.415 \mathrm{~V}$ which higher than the previous study with $2.058 \mathrm{~V}$. Roughly, it shows that the present study produced a better result. However, many factors may affect this result, thus, a difficult justification needs to be made at the end. The first component is the antenna. The previous study deployed a hybrid Kock Meander monopole antenna while in the present study, there is no particular antenna designed of antenna used, an AC supply was used to mimic the antenna instead. However, the present study was used an Omni-directional ducky antenna available on market as a reference.

As for the frequency band, both studies chose to use $2.4 \mathrm{GHz}$. This is because the Wi-Fi source has been the objective to be the source for the RF harvesting. Next for the rectifying method, the Cockcroft Walton voltage multiplier was chosen to be the rectifier circuit configuration. However, the different stage was recorded from both studies. The stages were recorded based on their ability to achieve their desired minimum voltage in each study. The minimum output voltage of $2.0 \mathrm{~V}$ was aimed from the previous study and $3.3 \mathrm{~V}$ was aimed in the present study. For that, 5 stages of the Cockcroft Walton circuit were recorded in the previous study while the present study recorded 3 stages to achieve their minimum desirable output voltage.

HSMS8260 was chosen by the previous study while BAT68 in the present study. The similarity shared between these two diodes is they both are Schottky type. Plenty of research in RF harvesting has chosen the Schottky diode because of its low forward voltage and high efficiency. Owing to Schottky diodes characteristics, it allows very switching speed with high accuracy which contribute a very big help for low power application with high frequency. This has been proved in the present study where BAT68 produces higher and better output than BAT17. But to compare between diodes in table 4.8, HSMS 8260 has a lower forward voltage compare to BAT68. However, due to the cost and availability of HSMS8260, BAT68 was chosen instead.

By logic reason of the characteristics between these diodes coupled with their rectifier stages, the output voltage recorded in the present study should have been lower than the previous study. On the other hand, the results recorded show contrarily. To justify the result, as stated before many factors may contribute to this discovery. The antenna is one of the main factors that may affect the result. By designing their antenna, its gained value, voltage standing wave ratio (VSWR), S-parameter and the impedance matching between the antenna and the rectifier are among the crucial parameter need to be decided carefully. All of these features can only be designed by using specific software such as Advanced Design System (ADS) by Keysight. Unfortunately, there is no accessibility for the software at the moment. Through Multisim, the close simulation that can be done is by replacing the antenna with a function generator which acts as the input source. The input source that has been used was based on the sinusoidal signals in the 50-Ohm system. This indicates that the power that has been transferred from the input into the rectifier system was at maximum efficiency. Unlike designing the antenna itself where the power transferred from source to rectifier will be based on the efficiency of the antenna design.

\section{CONCLUSION AND RECOMMENDATIONS}

To conclude, the objectives of this study have been achieved. However, there are many other factors can manipulate the output voltage. All of these factors should be included in the future study. Not to forget, since Schottky diode BAT68 has produced a promising result which is $4.415 \mathrm{~V}$, this diode should be considered to be used in the next study. All in all, a rectifier circuit on power over Wi-Fi for IoTbased sensor purposes was designed with the Cockcroft-Walton voltage multiplier configuration. Two types of Schottky diode were employed in the circuit. BAT68 has shown higher and better output compare to BAT17 for all stages. For stage one, the difference between these two diodes is $0.021 \mathrm{~V}$, where BAT68 final output was $1.438 \mathrm{~V}$ while BAT17 final output was $1.417 \mathrm{~V}$. For stage two, the difference recorded between the diodes is $0.039 \mathrm{~V}$ with the final output of $2.807 \mathrm{~V}$ from BAT68 and $2.768 \mathrm{~V}$ from BAT68. At stage three, the difference between diode BAT68 and BAT17 is $0.055 \mathrm{~V}$ with the final output of $4.145 \mathrm{~V}$ from BAT68 and $4.090 \mathrm{~V}$ from BAT17. For stage four, BAT68 final output was $5.464 \mathrm{~V}$ and BAT17 recorded its final output at $5.392 \mathrm{~V}$, resulting in the difference between this 
two on this stage at $0.0072 \mathrm{~V}$. Minimum voltage for IoT-based sensors also has been achieved and identified at stage 3 of the voltage multiplier.

Some improvements can be made in the future study. Among the improvements is a study on the antenna design that can be added in this study as it plays a very vital role in radio frequency energy harvesting. By adding the antenna study, the matching circuit can also be employed to match the impedance between the antenna and rectifier for maximum power transfer. The study on diodes used in energy harvesting also should not have only limited to Schottky types. How different type of diodes and rectifier configuration will affect the output should be done and studied.

\section{REFERENCES}

[1] Lenord Melvix, J. S. M., Lokesh, V. and Polyzos, G. C. "Energy efficient context based forwarding strategy in named data networking of things," ACM-ICN 2016 - Proc. 2016 3rd ACM Conf. Information-Centric Netw., pp. 249-254, 2016.

[2] Hong, S. S. B., Ibrahim, R., Khir, M. H. M., Daud, H. \& Zakariya, M. A. "Rectenna architecture based energy harvester for low power RFID application", 2012 4th International Conference on Intelligent and Advanced Systems (ICIAS 2012), June 12-14, 2012. https://doi.org/10.1109/ICIAS.2012.6306223

[3] Shafique, K., Khawaja, B.A., Khurram, M.D., Sibtain, S.M., Siddiqui, Y., Mustaqim, M., Chattha, H.T. \& Yang, X. "Energy Harvesting Using a Low-Cost Rectenna for Internet of Things (IoT) Applications," IEEE Access 6, 30932- 30941, 2018.

[4] Olgun, U., Chen, C.C., and Volakis, J. L. "Design of an efficient ambient WiFi energy harvesting system," IET Microwaves, Antennas Propag. 6(11), 1200-1206, 2012.

[5] Thosar, P. "Design of Rectenna using RF Harvesting for Batteryless IoT Sensors," Int. J. Futur. Revolut. Comput. Sci. Commun. Eng. 4(3), 63-67, 2018.

[6] Rosli, M. A., Murad, S. A. Z. and Ismail, R. C. "A 900-2400 MHz AC-DC Rectifier Circuit for Radio Frequency Energy Harvesting," MATEC Web Conf., vol. 78, 2016.

[7] Bandyopadhyay, P. and Dutta, D. "Wireless Energy Harvesting Using RF-Dc Module," IOSR Journal of Electrical and Electronics Engineering 13(4), 37-45, 2018.

[8] Bonaldi, E.L., Levy Ely de Lacerda de Oliveira, Germano Lamber-Torres, Luiz Eduardo Borges da Silva and Bernardes, V.A. "Current transducer for IoT applications," New Trends in the Use of Artificial Intelligence for the Industry 4.0, 2020. Available from: https://www.intechopen.com/books/new-trends-in-the-use-of-artificial-intelligence-for-theindustry-4-0/current-transducer-for-iot-applications

[9] Iqbal, M. S., Khanzada, T. J., Dahri, F. A., Ali, A., Ali, M. and Wahab, A. "Analysis and Maximizing Energy Harvesting from RF Signals using T-Shaped Microstrip Patch Antenna," International Journal of Advanced Computer Science and Applications 10(1), 2019. 